\title{
2019: A Sepsis Odyssey
}

\author{
Christine Cook MD, Steven Q Simpson MD, FCCP
}

\section{INTRODUCTION}

Sepsis, which comes from the Greek oń $\psi 1 \varsigma$, is not a new term. Rather, it was used as many as 2,700 years ago by Homer and, later, Hippocrates and Galen, to describe rotting flesh and the malodorous fluids and markers of inflammation that accompany biological decay. ${ }^{1,2}$ Throughout history, the medical community has been fascinated with the prevention, pathophysiology, and treatment of infection. Early recognition and treatment, after all, reduces morbidity and mortality. Efforts over the past several decades have focused on defining (and re-defining) sepsis, identifying early warning signs of its presence, and standardizing sepsis management to improve outcomes. Here we review the recent past, the controversial present, and the promising future of sepsis management.

\section{The Recent Past}

The first International Consensus Conference on Sepsis held in 1991 was a meeting of health care professionals representing the American College of Chest Physicians (ACCP) and the Society of Critical Care Medicine (SCCM) with the aim of standardizing the definition of sepsis and the approach to its treatment. The Consensus Conference committee adopted the phrase "systemic inflammatory response syndrome", or SIRS, to describe the physiologic response to infection and other non-infectious triggers of inflammation. Systemic inflammatory response syndrome requires that two or more of the following conditions be met: temperature $>38^{\circ} \mathrm{C}$ or $<36^{\circ} \mathrm{C}$; heart rate $>90$ beats per minute; respiratory rate $>20$ breaths per minute; and white blood cell count $>12,000 /$ cu $\mathrm{mm},<4,000 / \mathrm{cu} \mathrm{mm}$, or $>10 \%$ bands.

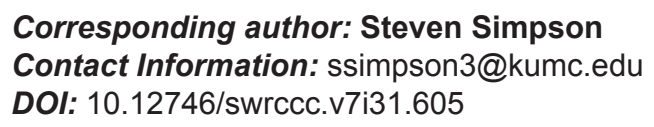

The SIRS criteria were meant to define sepsis-two positive SIRS criteria and the presence of infectionand to serve as a warning sign to prompt investigation for severe sepsis. Severe sepsis was defined as sepsis associated with organ dysfunction, hypoperfusion, or hypotension; septic shock was defined by sepsisinduced hypotension despite adequate fluid resuscitation, resulting in organ hypo-perfusion. ${ }^{3}$

Sepsis-2, the name given to the Second International Consensus Conference held in 2001, revisited these definitions but made no changes to its diagnostic criteria. Committee members representing the SCCM, the European Society of Intensive Care Medicine (ESICM), the ACCP, the American Thoracic Society (ATS), and the Surgical Infection Society (SIS) agreed with the previously established definitions. However, conference participants did conclude that the SIRS criteria were overly sensitive and not specific enough to sepsis. Therefore, other features that may be part of the robust inflammatory response seen in sepsis were identified as potential markers of sepsis and organ dysfunction. These features included altered mental status, elevated procalcitonin, ileus, thrombocytopenia, and mixed venous oxygen saturation (ScvO2) $>70 \%{ }^{4}$

In the years following Sepsis-2, efforts were made to standardize early treatment of sepsis using bundled care. The Surviving Sepsis Campaign, launched in 2002, introduced six- and 24-hour sepsis bundles. These bundles were revised in 2012 and became the three- and six-hour bundles that are commonplace today. Within three hours of presentation, patients presenting with possible sepsis should have a lactate level and blood cultures drawn, receive broad spectrum antibiotics, and be resuscitated with $20 \mathrm{~mL} / \mathrm{kg}$ (later increased to $30 \mathrm{~mL} / \mathrm{kg}$ ) of crystalloid fluid for hypotension or a lactate $>4 \mathrm{mmol} / \mathrm{L}$. Within six hours, vasopressors should be used to treat persistent hypotension, lactate should be rechecked, and ScvO2 and central venous pressure (CVP) should be used to help guide fluid resuscitation. ${ }^{5}$ 
With the adoption of sepsis bundles, resuscitation goals were established. Early goal-directed therapy (EGDT) was developed in a single-center study set in a busy urban emergency department. Rivers's seminal trial compared EGDT (control plus ScvO2 >70\%) to usual care with resuscitation goals of CVP 8 to $12 \mathrm{~mm} \mathrm{Hg}$, mean arterial pressure 65 to $90 \mathrm{~mm} \mathrm{Hg}$, and urinary output $>0.5 \mathrm{~mL} / \mathrm{kg} / \mathrm{hr}$ within the first six hours of presentation. The 28-day mortality rate for the EGDT arm was $33.3 \%$ compared to $49.2 \%$ in the usual care group. The more recent ProCESS (Protocol-Based Care for Early Septic Shock), ARISE (Australasian Resuscitation in Sepsis Evaluation), and ProMISe (Protocolized Management in Sepsis) randomized controlled trials (RCTs) looked at the impacts of EGDT on outcomes in larger sample sizes. In each of these studies, the differences in primary endpoints between the intervention and control groups were not statistically significant, perhaps because implementation of at least some aspects of EGDT has become standard care in many emergency departments and ICUs. ${ }^{6}$

These trials are often taken to indicate that EGDT is not useful; however, some weaknesses belie that notion, at least for some sepsis patients. The ProCESS, ARISE, and ProMISe trials were not blinded, and, therefore, a higher level of care may have been provided to control groups in these studies, potentially impacting outcomes. Furthermore, these studies evaluated all comers with septic shock, and, in the arms of the study where $\mathrm{ScvO}_{2}$ was measured, fully half of all patients had achieved the EGDT target $(70 \%)$ by the time of enrollment. Hence, the studies establish that not all patients with septic shock require EGDT but fail to tell us whether EGDT is appropriate or useful for patients who begin with poor tissue oxygenation evidenced by lower $\mathrm{ScvO}_{2}$. Moreover, the patient population in these trials was younger with fewer comorbidities and less need for mechanical ventilation when compared to the original EGDT trial. When actual hospital mortality of the ProCESS, ProMISe, and ARISE trials is subtracted from the APACHE II predicted mortality for each trial population, relative risk reduction in hospital mortality would be similar between the seminal EGDT trial and these more recent sepsis RCTs. ${ }^{6}$

\section{The Controversial Present}

Despite aggressive measures to identify sepsis early and manage it aggressively, sepsis remains a major public health concern and a drain on healthcare resources. A recent retrospective study found that more than $50 \%$ of adult hospitalizations ending in death or resulting in discharge to hospice were associated with sepsis. In fact, sepsis was identified as the immediate cause of death in more than two-thirds of these cases and was more common than progressive malignancy or heart failure. ${ }^{7}$

To help combat sepsis-associated mortality, rapid response teams and early warning systems have been implemented in hospitals around the world. Early warning system scores were designed to track patients at risk of deterioration and to trigger alarms before decompensation occurs. Early warning system scores perform well in predicting cardiac arrest and death within 48 hours but, overall, have not consistently been shown to reduce mortality or costs of care. ${ }^{8}$

In 2014, the Third International Consensus Conference convened to review the current understanding of sepsis. Sepsis-3 proposed a new definition of the term "sepsis": life-threatening organ dysfunction caused by a dysregulated host response to infection. The term "severe sepsis" was determined to be redundant and was removed from Sepsis-3 diagnostic criteria. The task force proposed the use of two tools to identify organ dysfunction and predict mortality. A score of two points or more on the Sequential Organ Failure Assessment (SOFA) indicates organ dysfunction. The SOFA score is intended for use in the ICU. The quick SOFA (qSOFA), which can be applied by clinicians at bedside on the wards, includes three criteria: respiratory rate $>22$ /minute, altered mentation, and systolic blood pressure $<100 \mathrm{~mm} \mathrm{Hg}$. If two of three criteria are met, the score is positive. Septic shock was redefined as sepsis with circulatory and cellular/metabolic abnormalities profound enough to substantially increase mortality. To meet the diagnostic criteria for septic shock according to Sepsis-3, patients must have a vasopressor requirement to maintain a mean 
arterial pressure $>65 \mathrm{~mm} \mathrm{Hg}$ and a lactate level $>2 \mathrm{mmol} / \mathrm{L}^{9}$

Verboom et al recently studied the robustness and discriminatory ability of the Sepsis-3 criteria in patients prospectively enrolled in the Molecular Diagnosis and Risk Stratification of Sepsis (MARS) cohort in the Netherlands. ${ }^{10}$ They found that minor variations in the interpretation of the SOFA criteria significantly affect the apparent prevalence of sepsis. For example, the initial Sepsis-3 paper by Singer et al defined organ failure as an acute change in the SOFA score of $\geq 2$ points, whereas follow-up validation studies used an absolute SOFA score of $\geq 2$. Minor variations in the application of the SOFA criteria (such as SOFA score increase vs. absolute SOFA score) can have a major impact on the apparent incidence of sepsis. The authors also found that $90 \%$ of patients with suspected infection upon ICU admission met Sepsis-3 criteria, compared to only $60 \%$ who met the MARS-sepsis criteria (presence of $\geq 2$ SIRS criteria and organ failure within a 4-day window around suspected infection). If nearly all patients with suspected infection admitted to the ICU meet Sepsis-3 criteria, distinguishing which patients will experience better or worse outcomes and why they will do so becomes a difficult task. ${ }^{10}$

When compared with SIRS, the SOFA and qSOFA scores are better able to predict mortality and prolonged ICU stay. But by the time organ dysfunction or multi-organ system failure is detected using the diagnostic criteria proposed in Sepsis-3, the opportunity to act early and prevent organ dysfunction has passed. The proverbial horse (organ dysfunction) is already out of the barn. In the age of Sepsis-3, the familiar three-hour and six-hour sepsis bundles have been combined into an aspirational "hour-1" bundle. ${ }^{11}$ This underscores the importance of immediacy in sepsis management. Resuscitation and management need to begin almost simultaneously with recognition of the problem. Early antibiotic administration in patients with severe sepsis reduces risk of progression to shock and mortality, and failure to meet early resuscitation goals increases 28 -day mortality. ${ }^{5,12}$ It is no surprise, then, that the recommended time window to deliver the sepsis bundle has been reduced to a single hour.

\section{The Promising Future}

Despite efforts to more clearly define and appropriately manage sepsis, its complexity and heterogeneity continue to challenge the healthcare community. Until now, sepsis has not been thought of as an illness amenable to staging, and no stages of sepsis comparable to the TNM stages of solid organ malignancy have been proposed. While sepsis may progress to shock, it does not necessarily take a one-lane, oneway street to get there. Patients may present for medical attention early in the course of their infection or late into their "dysregulated host response," and their organs may be in various states of dysfunction by the time they enter the healthcare system. Comorbidities, like renal dysfunction and liver failure, may impact host immune response and onset and progression of organ dysfunction.

We know that early recognition and treatment of sepsis saves lives. Bundled care and early warning systems have made their mark on sepsis management, but we live in an age of personalized medicine. How do we personalize the treatment of a heterogeneous entity like sepsis? As is the trend in other areas of medicine, sepsis researchers are turning their attention to novel biomarkers and phenotyping.

Historically, sepsis biomarkers have lacked timeliness and specificity. Blood cultures often take 24 to 48 hours to become positive, and C-reactive protein (CRP) must be synthesized by the liver in response to a pathogen before levels appear in serum hours later. Lactate is a non-specific marker for tissue hypoperfusion, but its rapid turnaround time and widespread availability are attractive. Moreover, lactate screening in sepsis has been shown to reduce mortality by $11 \%{ }^{6}$ Procalcitonin also has been evaluated as a biomarker in sepsis and can be a useful marker of response to therapy, resulting in decreased antibiotic use in the ICU. However, procalcitonin levels are also nonspecific and do not correlate with severity of sepsis. ${ }^{13}$ It is clear that early and specific biomarkers for sepsis are needed.

Numerous biomarkers of sepsis are actively under investigation. Presepsin is a soluble form of CD14 that is expressed on the membrane of macrophages and 
monocytes and indirectly stimulates release of TNFalpha. CD64 is an immunoglobulin receptor expressed on neutrophils with increased expression in response to cytokines in sepsis. Soluble-urokinase-typeplasminogen-activator-receptor (suPAR) is expressed on many immunologically active cells with upregulation in response to inflammation. Soluble triggering receptor expressed on myeloid cells 1 , or sTREM-1, is a receptor expressed on polymorphonuclear cells and mature monocytes with increased expression in a setting of bacterial or fungal infection. ${ }^{13}$ Additional biomarkers are under investigation, along with combinations of biomarkers and physiologic data. Machine learning and artificial intelligence are being applied to predict which patients are on a path to shock and multiple organ dysfunction and which are not.

Seymour et al recently published work deriving and validating four clinical phenotypes of sepsis. Their retrospective analysis included 16,552 unique patients who met Sepsis- 3 criteria within six hours of presentation to 12 Pennsylvania hospitals between 2010 and 2012. They applied consensus $\kappa$ means clustering to 29 variables and identified four phenotypes that correlated with host-response patterns and clinical outcomes. The $\delta$ phenotype was the least common but included patients with more liver dysfunction and shock and higher mortality. ${ }^{14} \mathrm{~A}$ separate study by Bhavani et al used group-based trajectory modeling to identify and validate temperature trajectory groups in patients admitted to the hospital with infection. Four temperature trajectory groups were identified: hyperthermic, slow resolvers; hyperthermic, fast resolvers; normothermic; and hypothermic. ${ }^{15}$ Better understanding of these sepsis phenotypes will likely allow us to unbundle and re-bundle sepsis treatment with a more personalized approach. Prescott and Iwashyna have proposed a pragmatic approach to personalizing care of the septic patient, based on the severity of illness and the degree of certainty that an infection is present. Patients with more evidence of organ dysfunction and in whom bacterial infection is more strongly suspected should be given empiric antibiotics as early as possible. For patients who are less ill and in whom symptoms of infection are more obscure, it may be appropriate to delay therapy until a more secure diagnosis can be established. ${ }^{16}$

\section{Conclusion}

The diagnostic criteria for sepsis and our approach to its management have evolved, but the disease itself has not. Early recognition and resuscitation remain the key tenets of survival. The incorporation of new biomarkers and machine learning hold great promise for even earlier recognition and appropriate intervention.

Keywords: sepsis, definition, diagnosis

Article citation: Cook C, Simpson SQ. 2019: A Sepsis Odyssey. The Southwest Respiratory and Critical Care Chronicles 2019;7(31):3-7

From: Department of Internal Medicine, University of Kansas School of Medicine, Kansas City, KS

Submitted: 9/26/2019

Accepted: 9/30/2019

Conflicts of interest: none

This work is licensed under a Creative Commons Attribution-ShareAlike 4.0 International License.

\section{REFERENCES}

1. Angus DC, van der Poll T. Severe sepsis and septic shock. N Engl J Med 2013;369(9):840-851.

2. Funk DJ, Parrillo JE, Kumar A. Sepsis and septic shock: A history. Crit Care Clin 2009;25:83-101.

3. Bone RC, Balk RA, Cerra FB, et al. Definitions for sepsis and organ failure and guidelines for the use of innovative therapies in sepsis. Chest 1992;101:1644-1655.

4. Levy MM, Fink MP, Marshall JC, et al. 2001 SCCM/ ESICM/ACCP/ATS/SIS International Sepsis Definitions Conference. Intensive Care Med 2003;29:530-538.

5. Zhang Z, Hong Y, Smischney NJ, et al. Early management of sepsis with early goal-directed therapy: AME evidence series 002. J Thorac Dis 2017;9(2):392-405.

6. Nguyen HB, Jaehne AK, Jayaprakash N, et al. Early goaldirected therapy in severe sepsis and septic shock: insights and comparisons to ProCESS, ProMISe, and ARISE. Critical Care 2016;20:160.

7. Rhee C, Jones TM, Hamad Y, et al. Prevalence, underlying causes, and preventability of sepsis-associated mortality in U.S. acute care hospitals. JAMA Network Open. Published online February 15, 2019.2(2):e187571. 
8. Smith ME, Chiovaro JC, O’Neil M, et al. Early warning system scores for clinical deterioration in hospitalized patients: a systematic review. Ann Am Thorac Soc 2014;11(9):1454-1465.

9. Singer M, Deutschman CS, Seymour CW, et al. The Third International Consensus definitions for sepsis and septic shock. JAMA 2016;315(8):801-810.

10. Verboom DM, Frencken JF, Ong D SY, et al. J Intensive Care. 2019;7:46.

11. Levy MM, Evans LE, Rhodes A. The Surviving Sepsis Campaign bundle: 2018 update. Intensive Care Med 2018;44:925-928.

12. Whiles BB, Deis AS, Simpson SQ. Increased time to initial antimicrobial administration is associated with progression to septic shock in severe sepsis patients. Crit Care Med 2017;45(4):623-629.
13. Larsen FF, Petersen JA. Novel biomarkers for sepsis: A narrative review. Eur J Intern Med 2017;45:46-50.

14. Seymour CW, Kennedy JN, Wang S, et al. Derivation, validation, and potential treatment implications of novel clinical phenotypes for sepsis. JAMA. Published online May 19, 2019.321(20):2003-2017.

15. Bhavani SV, Carey KA, Gilbert ER, et al. Identifying novel sepsis subphenotypes using temperature trajectories. Am J Respir Crit Care Med 2019;200(3):327-335. doi: 10.1164/ rccm.201806-11970C.

16. Prescott HC, Iwashyna TJ. Improving sepsis treatment by embracing diagnostic uncertainty. Ann Am Thorac Soc 2019; 16(4):426-429. 